\title{
Oral Health: Validation of a questionnaire of self-perception and self-care habits in Diabetes Mellitus 2, hypertensive and obese patients. The UISESS-B scale
}

\author{
Ana-Leticia Salcedo-Rocha ${ }^{1}$, Javier-Eduardo García-de-Alba-Garcia ${ }^{2}$, José-Gabriel Velásquez-Herrera ${ }^{3}$, \\ Elías-Alejandro Barba-González ${ }^{4}$
}

Correspondence:

Unidad de Investigación Social,

Epidemiológica y de Servicios de Salud,

Centro Médico de Occidente. (Bajos de la Farmacia Central),

Instituto Mexicano del Seguro Social.

Colonia Independencia, Guadalajara, Jalisco,

México CP 44340

javier_91046@yahoo.com

\author{
Salcedo-Rocha AL, García-de-Alba-Garcia JE, Velásquez-Herrera JG, \\ Barba-González EA. Oral Health: Validation of a questionnaire of self- \\ perception and self-care habits in Diabetes Mellitus 2, hypertensive and \\ obese patients. The UISESS-B scale. Med Oral Patol Oral Cir Bucal. 2011 \\ Sep 1;16 (6):e834-9. \\ http://www.medicinaoral.com/medoralfree01/v16i6/medoralv16i6p834.pdf \\ Article Number: $17108 \quad$ http://www.medicinaoral.com \\ (C) Medicina Oral S. L. C.I.F. B 96689336 - pISSN 1698-4447 - eISSN: 1698-6946 \\ eMail: medicina@medicinaoral.com \\ Indexed in: \\ Science Citation Index Expanded \\ Journal Citation Reports \\ Index Medicus, MEDLINE, PubMed \\ Scopus, Embase and Emcare \\ Indice Médico Español
}

\begin{abstract}
Objectives: To develop and to evaluate a questionnaire of self-perception and self-care habits on oral health on a first level population.

Methods: A descriptive, observational study was performed to validate a questionnaire on oral health self-perception (UISESS-B). After non-probabilistic sampling, 94 users, 30-59 years of age, with either diabetes mellitus 2, hypertension or obesity were included. Duration of disease was lesser than 10 years. Pearson's r, Cornbach's $\alpha$, factorial analysis, chi-square and Snedecor's F tests were employed.

Results: A Pearson's $r$ of 0.7 and Cronbach's $\alpha$ of 0.82 was observed on the pre-post values of the global questionnaire. In the factorial analysis, the variance explained more than $60 \%$ for a first factor. A punctuation of very high risk for the three groups with the scale UISESSS-B that coincides with the index CPO-D and the index UISESS-F was observed.

Conclusions: The UISESS-B scale shows significant validity and reliability, suggesting its use as a sensitive instrument for the measurement of oral health in people with chronic illnesses such as diabetes, hypertension and obesity.
\end{abstract}

Key words: Oral health questionnaire, diabetes, hypertension, obesity. 


\section{Introduction}

Oral diseases, such as cavities and periodontal disease, show an increasing prevalence during a person's life span (1), whereas its clinical treatment can be invasive and annoying for a lot of people. Most recently, due to ethical and legal issues (2), research on this topic has been oriented towards the use of questionnaires and interviews on the self-perception of oral health (3).

Data comparison among clinical examinations and self-perception questionnaires have shown the latter as good for determining oral health status and gingival conditions as well (4), with a near $50 \%$ sensitivity and sensibility (5). These questionnaires are primarily oriented towards clinical and single problems and explore the psychological and social dimensions of oral health's self-management (6).

The CPO-D scale is used worldwide to qualitatively evaluate cavities, lost or teeth with any other process in them, such a scale provides a one-time overview of oral health. Tools for the subjective evaluation of oral health are scarce in Mexico, one of them is the UISSES-F scale, which measures the oral functionality with a preventive focus (7). As for chronic diseases, oral health is regarded as one of many items accounting for a good quality of life (8). In Mexico, oral health evaluation is poorly included or not included at all in the diverse questionnaires focused on the evaluation of chronic diseases (diabetes, hypertension, obesity). In order to fill this gap we decided to develop and to evaluate a questionnaire termed the UISSES-B, which is focused on the self-evaluation of oral health in a first-level, medical-attention population (9).

\section{Methods}

An observational, descriptive study was used to evaluate a questionnaire on oral health self-perception. Nonprobabilistic sampling was used to recruit 94 patients from 2 different first-level of attention medical facilities in Guadalajara, Jalisco, Mexico. All of them were subjected to the inclusion criteria of 30-59 years of age and a medical diagnosis of one of the following diseases: diabetes, hypertension, obesity.

The questionnaire, termed UISSES-B Scale consists of two parts: 1) A sociodemographic evaluation and an institutional evaluation, 2) A self-perception oral health evaluation. This scale was elaborated as a questionnaire with the following work-up:

1. A first questionnaire was done by the authors based on the knowledge of the basic oral health-disease process and on the basis of prevention.

2. Validation, performed by a group of experts, unrelated to the development of the questionnaire. A blind, Delphi technique was employed in order to validate the questionnaire and consisted of having a group of experts (1 dentist, 1 epidemiologist, 1 social sciences re- searcher and 1 health services researches) with at least 10 years of experience in their respective field to analyze the scales and items in terms of the referred objectives. They were also asked to approve, modify or suggest new items. Both the experts and the authors discussed and approved the logical value of the proposed items.

3. A pilot study was done in 30 people from dental units in a first-level of medical attention in order to detect and to correct any caveat in a preliminary version of the questionnaire. Special attention was applied on the legibility and comprehension of the contents of each question. Each patient was asked to answer and comment the lecture easiness (very easy, easy, regular, difficult, very difficult) and the comprehension (very comprehensible, comprehensible, poorly comprehensible, not comprehensible) (10). No modifications were performed, for all of the subjects reported an easiness and comprehensibility of the questionnaire.

4. The final version see (Table 1) consisted of a selfadministered questionnaire divided in 2 subscales:

A) Oral health habits, composed of 32 items on self care:

- Hygiene (9 items)

- Food (9 items)

- Protection (14 items)

All of which were then subdivided in 3 categories concerning the frequency of the habit: always, sometimes, never.

B) Oral health status self-perception, composed of 16 items:

- Mouth and teeth signs

- Mouth and teeth symptoms

All of which had 3 categories concerning the frequency of presentation: never, sometimes, frequent.

5. The questionnaire was applied by one of the researchers (a dentist) to 94 people who had previously received indications on how to fill it in. Each subject filled the questionnaire in as a pre-test, followed 3 weeks later as a post-test (in order to avoid remembering the items of the test).

Data was processed on SPSS 15 and analyzed by means of averages and standard deviation for numerical data. In order to compare pre and post-test data, Cronbach $\alpha$, intercorrelation and Pearson's test (the latter of which was also used to validate the design of the study, to compare UISSES-B with the CPO-D index and with the UISSES-F index, which were also assessed on the subjects). A factorial analysis was also performed in order to evaluate the structure of the questionnaire. Snedecor's $F$ test was used to evaluate the internal structure of the questionnaire, whereas a Chi-square was used for analyzing categorical data. A p $\leq 0.05$ was used for statistical significance. 
Table 1. Initial and Final Instrument UISESSS- B scale (BUCO-DENTAL).

\begin{tabular}{|c|c|c|c|c|}
\hline Scale & \multirow{2}{*}{ Question: } & \multirow{2}{*}{ Always } & \multirow{2}{*}{ Sometimes } & \multirow{2}{*}{ Never } \\
\hline Habits & & & & \\
\hline \multirow[t]{10}{*}{ Feeding } & Value in point & $(3)$ & $(2)$ & $(1)$ \\
\hline & I eating irritating food (deleted) & & & \\
\hline & I eating hard food (deleted) & & & \\
\hline & Usually take refreshments & & & \\
\hline & I love eating chocolates & & & \\
\hline & I love salty and fatty foods as snacks & & & \\
\hline & I need to eat something between meals & & & \\
\hline & I take much water to avoid dryness $*$ & & & \\
\hline & I eat to my hours* & & & \\
\hline & I eat anytime & & & \\
\hline \multirow[t]{10}{*}{ Hygiene } & Value in point & $(1)$ & $(2)$ & $(3)$ \\
\hline & I wash my teeth after eating (deleted) & & & \\
\hline & Use revealing pill (deleted) & & & \\
\hline & Use mouthwash & & & \\
\hline & I wash my teeth to get up and go to bed & & & \\
\hline & Brush my tongue & & & \\
\hline & Use dental floss & & & \\
\hline & Change of toothbrush & & & \\
\hline & I massage my gums & & & \\
\hline & I wash my teeth after meals & & & \\
\hline \multirow[t]{14}{*}{ Care } & Value in point & (3) & $(2)$ & $(1)$ \\
\hline & Avoid taking things that pasted me (deleted) & & & \\
\hline & I reviewed my mouth to mirror frequently $*$ & & & \\
\hline & I'm going to the dentist to review even without problems * & & & \\
\hline & I Usually chew gum & & & \\
\hline & I smoke & & & \\
\hline & I use my teeth to peel things & & & \\
\hline & Use toothpicks to get me food & & & \\
\hline & I bite my nails & & & \\
\hline & Chattering teeth & & & \\
\hline & Chewing ice & & & \\
\hline & how things not too cold or too hot & & & \\
\hline & I usually get me things in their mouths as the pencil & & & \\
\hline & chattering teeth when I sleep & & & \\
\hline Perceptions & Question: & Not for nothing & Sometimes & Always \\
\hline \multirow[t]{6}{*}{ Symptoms } & Value in point & $(3)$ & $(2)$ & $(1)$ \\
\hline & I have pain to bite (deleted) & & & \\
\hline & I have bad taste in the mouth & & & \\
\hline & I have bad breath & & & \\
\hline & I have red gums & & & \\
\hline & I have sensitive teeth & & & \\
\hline \multirow[t]{12}{*}{ Signs } & Value in point & (3) & $(2)$ & $(1)$ \\
\hline & I have constant pain in the teeth (deleted) & & & \\
\hline & I have loss of teeth (deleted) & & & \\
\hline & I have abscesses (deleted) & & & \\
\hline & I have plaque buildup (deleted) & & & \\
\hline & I have cough with phlegm (deleted) & & & \\
\hline & I have thrush (deleted) & & & \\
\hline & I have bleeding gums & & & \\
\hline & I have loose teeth & & & \\
\hline & I have fires & & & \\
\hline & I have ulcers & & & \\
\hline & I have bruised gums & & & \\
\hline
\end{tabular}


Table 2. Main characteristics of the Group and groups of patients studied.

\begin{tabular}{|c|c|c|c|c|c|c|}
\hline \multirow{2}{*}{\multicolumn{2}{|c|}{ Feature }} & Diabetics & Hypertensive & Obese & Total & \multirow{2}{*}{ Results } \\
\hline & & $n=16$ & $n=28$ & $\mathrm{n}=\mathbf{5 0}$ & $n=94$ & \\
\hline \multirow{2}{*}{ Sex } & Male & $25 \%$ & $43 \%$ & $22 \%$ & $24 \%$ & \multirow{2}{*}{$\begin{array}{c}\mathrm{Ji}^{2}=3.94 \\
\mathrm{p}=0.13\end{array}$} \\
\hline & Female & $75 \%$ & $57 \%$ & $78 \%$ & $76 \%$ & \\
\hline Age & Average & $\begin{array}{c}51.81 \pm 5.0 \\
8\end{array}$ & $52.04 \pm 6.27$ & $46.88 \pm 8.66$ & $47.9 \pm 8.13$ & $\begin{array}{l}F=5.36 \\
p=0.006\end{array}$ \\
\hline Schooling & Average & $7.62 \pm 4.92$ & $9.46 \pm 5.35$ & $8.60 \pm 4.22$ & $8.82 \pm 4.58$ & $\begin{array}{c}\mathrm{F}=0.80 \\
\mathrm{p}=0.45\end{array}$ \\
\hline \multirow[t]{2}{*}{ Activity } & Worker & $44 \%$ & $46 \%$ & $48 \%$ & $47 \%$ & \multirow{2}{*}{$\begin{array}{c}\mathrm{Ji}^{2}=0.17 \\
\mathrm{P}=0.67\end{array}$} \\
\hline & Home & $56 \%$ & $54 \%$ & $52 \%$ & $53 \%$ & \\
\hline $\begin{array}{c}\text { History } \\
\text { Diagnostic }\end{array}$ & Average & $5.87 \pm 6.25$ & $7.12 \pm 6.13$ & $10.35 \pm 8.54$ & $7.14 \pm 6.68$ & $\begin{array}{l}F=2.93 \\
p=0.06\end{array}$ \\
\hline
\end{tabular}

Table 3. Pre-post test scores, correlations and alphas found in U-B subscale (scale UISESSS-B)

\begin{tabular}{|c|c|c|c|c|}
\hline \multicolumn{5}{|l|}{ Habits } \\
\hline $\begin{array}{c}\text { Phase } \\
\text { Domain }\end{array}$ & Feeding & Hygiene & Care & Totals \\
\hline Average pre test & $23.10 \pm 3.22$ & $14.12 \pm 3.46$ & $30.55 \pm 6.43$ & $67.54 \pm 7.87$ \\
\hline Average post test & $23.94 \pm 3.45$ & $17.76 \pm 3.3$ & $29.35 \pm 2.99$ & $70.19 \pm 6.60$ \\
\hline $\mathrm{P}$ & 0.56 & 0.75 & 0.55 & 0.82 \\
\hline r-Pearson & 0.610 & 0.733 & 0.651 & 0.706 \\
\hline Crombach Alpha & 0.796 & 0.844 & 0.778 & 0.822 \\
\hline $\mathrm{r}$ intraclase & 0.661 & 0.730 & 0.637 & 0.698 \\
\hline $\mathrm{P}$ & 0.05 & 0.002 & $<0.001$ & $<0.001$ \\
\hline \multicolumn{5}{|l|}{ Perceptions } \\
\hline $\begin{array}{c}\text { Phase } \\
\text { Domain }\end{array}$ & Signs & Symptoms & Totals & \\
\hline Average pre test & $11.52 \pm 2.05$ & $29.12 \pm 3.05$ & $40.66 \pm 4.54$ & \\
\hline Average post test & $12.15 \pm 1.93$ & $30.10 \pm 2.86$ & $42.52 \pm 4.00$ & \\
\hline $\mathrm{P}$ & 0.61 & 0.59 & 0.29 & \\
\hline r-Pearson & 0.725 & 0.644 & 0.745 & \\
\hline Crombach Alpha & 0.838 & 0.777 & 0.849 & \\
\hline $\mathrm{r}$ intraclase & 0.636 & 0.682 & 0.738 & \\
\hline $\mathrm{P}$ & $<0.001$ & $<0.001$ & $<0.001$ & \\
\hline
\end{tabular}

\section{Results}

A majority of the subjects were female, between the $4^{\text {th }}$ and $5^{\text {th }}$ decade of life, with 9 years of education and duration of disease of 5 to 9 years. Diabetics and obese are predominantly female subjects $(\mathrm{p}=0.13)$. No statistical difference was found in terms of education $(p=0-45)$, activity $(p=0.17)$ and duration of disease $(p=0.06)$, even though obese subjects had a longer duration. Only age was statistically significant $(\mathrm{p}=0.006)$ see (Table 2$)$.

Means of the pre and post evaluations on the habits and self-perceptions subscales were not statistically significant, contrary to the pre and post of total evaluations and those of Cronbach's $\alpha$.

On the habits subscale of the final questionnaire, the factorial analysis showed that, a first arrangement of the items concerning food, hygiene and care, explains
$68.85 \%$ of its variability $(\mathrm{p}<0.001)$. For the self-perception subscale (which included signs and symptoms), the first arrangement explained $62.29 \%$ of the variability $(\mathrm{p}<0.01)$. (Table 3$)$ shows:

1. The UISESS-B scale shows very high risk values for all of the groups.

2. The CPO-D index assigns all of the groups to a very high severity, when means are taken into account.

3. The UISESS-F index assigns all diabetic patients into a very high degree of dysfunctionality and the hypertensive into a high degree, when means are taken into account.

The highest correlation between the CPO-D and the UISSES-B indexes lies among de diabetic patients. (Table 4) shows the final version of the questionnaire, noting the 12 items of the original version that were 
Table 4. Average score and percentage of risk as: Scale UISESS-B, Index CPO and Index UISESS -F by patient groups.

\begin{tabular}{|c|c|c|c|c|c|}
\hline \multirow{3}{*}{ Concept } & Diabetic & \multirow{3}{*}{$\begin{array}{c}\text { Hypertensions } \\
\mathrm{n}=28 \\
\text { Average } \\
(\% \text { risk higher })\end{array}$} & Obese & \multirow{2}{*}{\multicolumn{2}{|c|}{$\begin{array}{c}\text { Analysis } \\
\text { intergroup }\end{array}$}} \\
\hline & $\mathrm{n}=16$ & & \multirow{2}{*}{$\begin{array}{c}\mathrm{n}=50 \\
\text { Average } \\
(\% \text { risk higher })\end{array}$} & & \\
\hline & $\begin{array}{c}\text { Average } \\
\text { (\% risk higher) }\end{array}$ & & & $\mathbf{F}$ & $\mathbf{P}$ \\
\hline UISESS-B & $111.85 \pm 9.37(100 \%)$ & $113.25 \pm 8.08(100 \%)$ & $113.16 \pm 9.63(100 \%)$ & 0.40 & 0.67 \\
\hline I. CPO-D & $16.63 \pm 1.87 \quad(88 \%)$ & $14.29 \pm 3.68 \quad(71 \%)$ & $12.94 \pm 5.64 \quad(92 \%)$ & 3.89 & 0.02 \\
\hline I UISESS- F & $7.77 \pm 5.09 \quad(56 \%)$ & $5.48 \pm 3.43 \quad(33 \%)$ & $5.21 \pm 3.17 \quad(30 \%)$ & 2.91 & 0.05 \\
\hline $\mathrm{r}$ of Pearson & $0.87 \mathrm{p}<0.000$ & $0.66 \mathrm{p}<0.00$ & $0.70 \mathrm{p}<0.00$ & & \\
\hline
\end{tabular}

eliminated due to its low response frequency or of correlation to the total scale: 5 from the habits subscale, 2 concerning the food, 2 for hygiene and 1 for care; from the self-perception subscale, 6 were related to signs and 1 for symptoms.

\section{Discussion}

Data obtained reveal the validity of a questionnaire where a prospective sampling was considered the best choice, due to the difficulty in finding people with a single disease. This was done: a) to avoid co-morbidity, so frequent in these diseases thus reducing any methodological error; b) because the objective was to develop and test a questionnaire and not to obtain causal relations.

All of the groups had no statistical differences among sex, age, education and duration of disease, which makes them a more homogenous population in a first-level of medical attention (which provides medical services to the middle and lower urban economical strata).

The UISSES-B finds its content validation in the review from the group op experts, the legal results and the acceptance obtained in the pilot study. The reliability of the global scale and the 2 subscales was shown by the consistency on the mean punctuations in the pre and post, with no statistically significant differences (6); correlations on the pre and post global scales over 0.7 and the statistically significant values on the Cronbach $\alpha$ test (equal or superior to 0.77 ).

The components on each subscale and the global scale showed internal validity by showing a solid factorial model with significant explaining variances (some questions were previously eliminated), making inconsistent the rotation of the studied factors. On all of the groups, the global punctuations correspond to a very high risk level in both the UISSES-B scale and the CPO-D index and the UISSES-F (7). As for the CPO-D, lower values than those observed in Brazil were found (25-31) for older patients (11).

The higher punctuation on the UISSES-B scale on the diabetic patients shows a high sensibility on the ques- tionnaire in patients whose hyperglycaemia (and other factors) can alter the oral flora (12) and predispose the emergence of the alterations assessed on the questionnaire that, when added up, increase the scale's punctuation.

During the selection of the items, the UISSES-B scale was reduced to 35 of them, diminishing the time of application, which we consider aids on the surveying of a population, limited to a first-level of attention, where a chronic lack of technical and professional dental human resources degrades the capacity for examination with the basic equipment needed for oral health examinations in the CPO or other indexes. This in order to properly diagnose the dental status such as: the O'Leary for bacterial plaque, Löe and Silness' gingival, OHI-S for oral hygiene, the CPTIN for paraodontal communitarian treatment and the UISSES-F functionality index $(7,13)$

The usefulness of the scale on the population with chronic disease is paramount for diabetes mellitus 2, hypertensive and obese patients (7) on a first-level basis, considering these are clusters of institutionalized subjects with a high impact on the health system and because the first two diseases are considered of high risk for oral health (14).

In populations such as ours (latin-american), with a high prevalence of oral health problems and with a long-lasting lack of human resources and of equipment in institutionalized health facilities, it is necessary to increase the efficiency of the institutions and focus primarily on the adult population, specially in those with a metabolic syndrome (15) or with any of their components, most of all because of the possibility of avoiding any of the complications of a chronic disease, by preventing periodontal disease (16).

Self-perception scales have a lower impact then the oral health examination, that is why self-perception is complemented, in the UISSES-B scale, with the subset of observable hygienic measures. For such reason, the UISSES-B scale can constitute a questionnaire that aids in the amelioration of the quality of life (17), to fill in the 
necessity of making people more conscious on chronic ailments with higher risk for oral health (18), to aid in the prevention by focusing on early diagnoses of risk factors, early treatment of dental diseases and by means of a screening test for the initiation of dental attention, for neither specialized equipment nor human resources is necessary for the surveying. We are nevertheless aware that a validation and reliability assessment on other populations is mandatory.

\section{Conclusión}

The significant levels of validation and reliability shown by the UISSES-B scale prompt it as a sensible questionnaire for the measurement of oral health risk in people with chronic diseases such as diabetes, hypertension and obesity.

\section{References}

References with links to Crossref - DOI

1. Barmes DE. A global view of oral diseases: today and tomorrow. Community Dent Oral Epidemiol. 1999;27:2-7.

2. Robinson PG, Nadanovsky P, Sheiham A. Can questionnaires replace clinical surveys to assess dental treatment needs of adults? J Public Health Dent. 1998;58:250-3.

3. Gilbert AD, Nuttall NM. Self-reporting of periodontal health status. Br Dent J. 1999;186:241-4.

4. Heloë LA. Comparison of dental health data obtained from questionnaires, interviews and clinical examination. Scand J Dent Res. 1972;80:495-9.

5. Tervonen T, Knuuttila M. Awareness of dental disorders and discrepancy between "objective" and "subjective" dental treatment needs. Community Dent Oral Epidemiol. 1988;16:345-8.

6. Montero-Martín J, Bravo-Pérez M, Albaladejo-Martínez A, Hernández-Martín LA, Rosel-Gallardo EM. Validation the Oral Health Impact Profile (OHIP-14sp) for adults in Spain. Med Oral Patol Oral Cir Bucal. 2009;14:E44-50.

7. Salcedo-Rocha AL, Sánchez-Mar M, López-Pérez Mde L, Preciado-Soltero HT, Figueroa-Suárez PA, Narváez-Aguirre VA, et al. Oral signs in patients with type-2 diabetes, hypertension and obesity. Rev Med Inst Mex Seguro Soc. 2006;44:203-10.

8. López-Carmona JM, Ariza-Andraca CR, Rodríguez-Moctezuma JR, Munguía-Miranda C. Development and initial validation of an instrument to measure the lifestyles of type 2 diabetes mellitus patients 2. Salud Publica Mex. 2003;45:259-68.

9. Rosas Peralta M, Lara Esqueda A, Pastelín Hernández G, Velázquez Monroy O, Martínez Reding J, Méndez Ortiz A, et al. National Re-survey of Arterial Hypertension (RENAHTA). Mexican consolidation of the cardiovascular risk factors. national follow-up cohort]. Arch Cardiol Mex. 2005;75:96-111.

10. Jung SH, Ryu JI, Tsakos G, Sheiham A. A Korean version of the Oral Impacts on Daily Performances (OIDP) scale in elderly populations: validity, reliability and prevalence. Health Qual Life Outcomes. 2008;6:17.

11. Moreira Rda S, Nico LS, Tomita NE, Ruiz T. Oral health of Brazilian elderly: a systematic review of epidemiologic status and dental care access. Cad Saude Publica. 2005;21:1665-75.

12. Do Amaral FM, Ramos PG, Ferreira SR. Study on the frequency of caries and associated factors in type 1 diabetes mellitus. Arq Bras Endocrinol Metabol. 2006;50:515-22.

13. Payette M, Brodeur JM. Comparison of dental caries and oral hygiene indices for 13- to 14-year-old Quebec children between 1977 and 1989-1990. J Can Dent Assoc. 1992;58:921-2, 926-9, 932-3.

14. Beck JD, Offenbacher S. Systemic effects of periodontitis: epidemiology of periodontal disease and cardiovascular disease. J Periodontol. 2005;76:2089-100.
15. Morita T, Ogawa Y, Takada K, Nishinoue N, Sasaki Y, Motohashi $\mathrm{M}$, et al. Association between periodontal disease and metabolic syndrome. J Public Health Dent. 2009;69:248-53.

16. Morita T, Yamazaki Y, Mita A, Takada K, Seto M, Nishinoue $\mathrm{N}$, et al. A cohort study on the association between periodontal disease and the development of metabolic syndrome. J Periodontol. 2010;81:512-9.

17. Montero J, Bravo M, Albaladejo A. Validation of two complementary oral-health related quality of life indicators (OIDP and OSS 0-10 ) in two qualitatively distinct samples of the Spanish population. Health Qual Life Outcomes. 2008;6:101.

18. Bullon P, Morillo JM, Ramirez-Tortosa MC, Quiles JL, Newman $\mathrm{HN}$, Battino M. Metabolic syndrome and periodontitis: is oxidative stress a common link? J Dent Res. 2009;88:503-18. 